\title{
Preprint servers to enhance access to scientific knowledge
}

Khaled Moustafa*

* Manager of FrenXiv

Email: khaled.moustafa@frenxiv.org

\begin{abstract}
Preprint servers can enhance the access to scientific knowledge by linking/indexing papers in bibliography databases such as Google scholar, PubMed, Web of Science, etc., to their counterpart preprint versions whenever available. The current state of connection/indexation is to link preprints to their published versions in peer-reviewed journals. Here, I suggest the opposite. That is, linking or indexing journal papers to their preprint versions wherever these are posted on a preprint server. Such linking from paid versions (journals' articles) to their corresponding free preprint versions would make much sense as it removes barriers to get access to paywall papers for free. Ideally, preprints should be indexable/discoverable in major bibliography indexers, but linking peer-reviewed versions to their preprints would increase access and sharing of scientific knowledge.
\end{abstract}

Keywords: preprint servers; article processing charge (APC); open access; scientific literature indexation; journal publication; journal publishing;

Scientific knowledge is a common heritage for humanity. It contributes to the well-being of humans and improves the interaction of people with each other and with their surrounding environments. Restricting access to scientific information can slow down innovation and hinder research to develop effective solutions to current and future challenges. Opening up science to be freely accessible and sharable is one of the most efficient ways to advance scientific knowledge and to solve problems related to health, environment, and society. Science should not only be open but also free ${ }^{1}$ as open science does not always means free of charge but often it involves high publication fees that many small to med-sized laboratories cannot afford.

Preprint servers are one of the effective tools to achieve such goals. Posting manuscripts in online repositories is commonplace in physics and mathematics since decades ago and the number of preprint servers in different disciplines/languages has grown over the few past years. The value of preprints to accelerate science communication is increasingly recognized and approved by scientific communities and evaluation committees. Preprints offer many advantages to scientists by making their thoughts freely and swiftly available. Preprints also help authors establish priority through date stamp to protect their thoughts and findings 
against scooping. Most traditional journals and publishers including Springer, Elsevier, Taylors and Francis, Wiley currently encourage authors to share their manuscripts as preprint versions before and/or after their manuscripts are peer-reviewed.

Once a paper is formally published in a journal, the authors are advised to link their preprint versions to the final journal-published versions. This is a useful endeavor to inform readers that the findings and ideas reported in a preprint version have passed the peer-review stage and are now available in a peer-reviewed journal. However, when the publishing journal is pay walled, which is mostly the case, linking a free preprint version to its paywall version does not help much as the paper will be unavailable for readers because of a paywall or subscription requirement. Therefore, I think that the opposite scenario would be more helpful. That is, linking a paid journal's paper to its free preprint version would make much sense than pointing out a free preprint version to its counterpart journal's paid version.

Thus, to facilitate open access to knowledge via preprint servers, I suggest that major bibliography indexers such as PubMed, PubMed Central, Google Scholar, etc., can index preprints or at least add links for indexed articles to their corresponding preprint versions, whenever available in a given preprint server. Google scholar already does this job to some extent but not yet fully optimized. PubMed does not yet offer such connection. As preprint versions are mostly assigned to digital object identifiers (DOI), linking journal published articles to their preprints versions, if available, would relatively be easy. PubMed central sometimes connect papers to their journals' free versions when publishing journals are open access, through links near articles' titles stating that a free full text is available (Figure. 1).

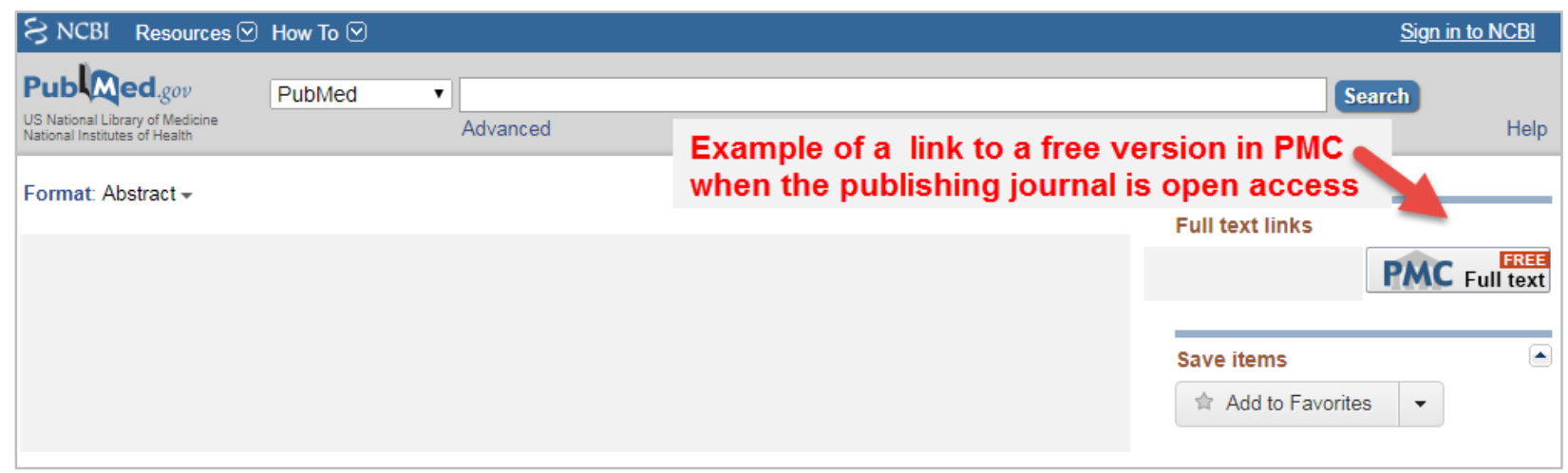

Figure 1. An example of free full text in PubMed Central when an article is published in open access journal or funded by a grant from the National Institute of Health (NIH).

In the same way, it would be of high interest to link paywall journals' articles indexed in PubMed to their counterpart preprint versions wherever posted on a preprint server (Figure 2). This would reinforce the discoverability and the value of preprint servers in boosting open 
access movement and to help readers get free access to scientific contributions transparently and legitimately.

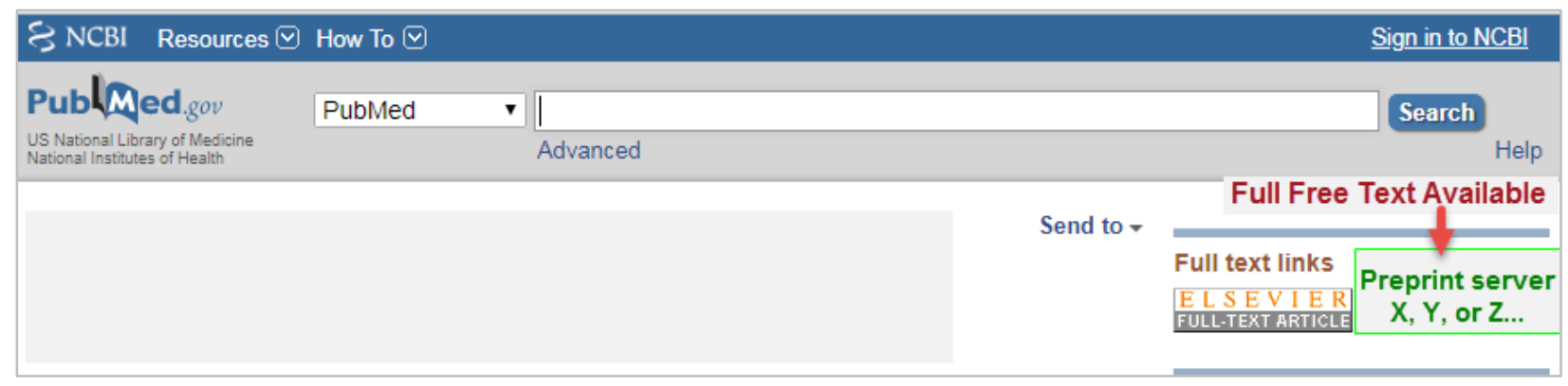

Figure 2. An example of potential linking of an indexed paper in PubMed to a preprint version posted in a given preprint server (X, Y, or Z...)

Ideally, preprints posted on preprint servers should be linked or indexed in major bibliography indexers, such as Google Scholar, Web of Science, PubMed/PubMed Central, etc., as they can be a great source of information particularly in times of environment or health crises, as is currently the case with corona virus disease 19 (COVID-10) where sharing knowledge through preprints was effective and popular.

Indexing preprints in bibliography indexers by pointing out readers from paid versions- journal articles- to free versions- preprints- as suggested here, is more logical than to point out readers from free preprint versions to paid journal versions where many authors in low resource economies may not be able to afford high costs of publishing and/or reading.

\section{References}

$1 \quad$ Moustafa, K. (2015). "Open access, open business, closed fairness!" Accountability in research 22, 246-248, doi:10.1080/08989621.2014.989220. 